\title{
An ECM-based energy-efficiency optimization approach for bandwidth-limited streaming kernels on recent Intel Xeon processors
}

\author{
Johannes Hofmann \\ Department of Computer Science \\ University of Erlangen-Nuremberg \\ Erlangen, Germany \\ johannes.hofmann@fau.de
}

\author{
Dietmar Fey \\ Department of Computer Science \\ University of Erlangen-Nuremberg \\ Erlangen, Germany \\ dietmar.fey@fau.de
}

\begin{abstract}
We investigate an approach that uses low-level analysis and the execution-cache-memory (ECM) performance model in combination with tuning of hardware parameters to lower energy requirements of memory-bound applications. The ECM model is extended appropriately to deal with software optimizations such as nontemporal stores. Using incremental steps and the ECM model, we analytically quantify the impact of various single-core optimizations and pinpoint microarchitectural improvements that are relevant to energy consumption. Using a 2D Jacobi solver as example that can serve as a blueprint for other memory-bound applications, we evaluate our approach on the four most recent Intel Xeon E5 processors (Sandy Bridge-EP, Ivy BridgeEP, Haswell-EP, and Broadwell-EP). We find that chip energy consumption can be reduced in the range of $2.0-$ $2.4 \times$ on the examined processors.
\end{abstract}

\section{Keywords}

ECM; 2D Jacobi; Performance Engineering; Energy Optimization

\section{INTRODUCTION AND REL. WORK}

For new HPC installations contribution of power usage to total system cost has been increasing steadily over the past years 10 and studies project this trend to continue [7]. As a consequence energy-aware metrics have recently been gaining popularity. Energy-tosolution, i.e. the amount of energy consumed by a system to solve a given problem, is the most obvious

Permission to make digital or hard copies of all or part of this work for personal or classroom use is granted without fee provided that copies are not made or distributed for profit or commercial advantage and that copies bear this notice and the full citation on the first page. Copyrights for components of this work owned by others than ACM must be honored. Abstracting with credit is permitted. To copy otherwise, or republish, to post on servers or to redistribute to lists, requires prior specific permission and/or a fee. Request permissions from permissions@acm.org.

E2SC '16 November 14 ${ }^{\text {th }}$, 2016, Salt Lake City, UT, USA

( 2016 ACM. ISBN $123-4567-24-567 / 08 / 06 \ldots \$ 15.00$

DOI: $10.475 / 123 \_4$ of these metrics and will be used as quality measure throughout this study.

Previous works view the application code as constant and instead focus their energy optimization attempts to parameter tuning on either the runtime environment, the hardware, or both [11]. The runtime environment approach works by adjusting the number of active threads across different parallel regions based on the regions' computational requirements 11. Hardware parameter tuning involves trying to identify slack, e.g. during MPI communication, and using dynamic voltage and frequency scaling (DVFS) to lower energy consumption in such sections of low computational intensity [14]; similar strategies can be applied to OpenMP barriers 2. The parameters employed by these algorithms can be based on measurements made at runtime 17] or set statically [16]. One fact often ignored by DVFS control software, however, is that hardware delays caused by changing frequency states can often be significant 12 which can lead to diminishing returns in the real world. Another form of hardware parameter optimization involves the tuning of hardware prefetchers 21 .

In contrast to previous work, our approach focuses on increasing single-core performance, primarily though software optimization. Using the execution-cachememory (ECM) performance model to guide optimizations such as SIMD vectorization, cache blocking, nontemporal stores, and the use of cluster-on-die (COD) mode the bandwidth consumption of a single core is maximized. This allows the chip to saturate main memory bandwidth with the least number of active cores in the multi-core scenario, thus reducing power as well as the energy consumed by the chip. In a second step, different hardware parameters such as the number of active cores and their frequencies should be evaluated to further reduce energy consumption. The approach is demonstrated on a 2D Jacobi solver, which acts as a proxy for many memory-bound applications. To produce significant results, all experiments were performed on the four most recent server microarchitectures by Intel, which make up about $91 \%$ of HPC installations of the June 2016 Top 500 list.

The paper is organized as follows. Section 2 describes 

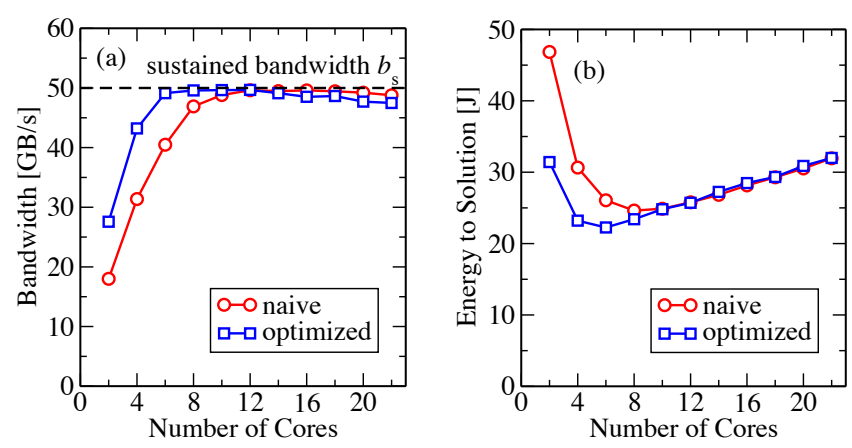

Figure 1: Bandwidth and energy-to-solution for different 2D Jacobi implementations using a dataset size of 16 GB.

the blueprint of and the reasoning behind our energyefficiency optimization approach. Section 3 introduces the ECM performance model that we use to guide optimizations. Section 4 contains an overview of the systems used for benchmarking. Core-level improvement efforts are documented in Section 5 . followed by a validation of single-core results in Section 6. Implications for the multi-core scenario are discussed in Section 7. followed by the conclusion in Section 8

\section{OPTIMIZATION APPROACH}

Per definition, the bottleneck for bandwidth-bound applications is the sustained bandwidth $b_{\mathrm{s}}$ imposed by the memory subsystem. It is a well-established fact that a single core of modern multi- and many-core systems cannot saturate main memory bandwidth [19], necessitating the use of multiple cores to reach peak performance. The number of cores $n_{\mathrm{s}}$ required to sustain main memory bandwidth depends on single-core performance: The faster a single-core implementation, the more bandwidth is consumed by a single core. The more bandwidth is consumed by a single core, the fewer cores are required to saturate main memory bandwidth.

This relation is illustrated in Fig. 1] which depicts measurement results obtained on a single socket of a standard two-socket Broadwell-EP machine (cf. Section 4 for hardware details). Fig. 1 1 shows memory bandwidth as function of active cores for two different 2D Jacobi implementations. Fig. $1 \mathrm{~b}$ depicts the energy-to-solution required for both implementations. From this real-world example two important conclusion can be drawn:

\section{Memory-bound codes should be run using $n_{\mathrm{s}}$ cores} Once the bandwidth bottleneck is hit, adding more cores no longer increases performance ${ }^{1}$ Instead using more cores increases chip power consumption resulting in a higher energy-to-solution. This effect is visible in Fig. 1p.

2. Early saturation can lead to lower energy-to-solution The optimized version saturates memory bandwidth with six cores compared to the ten cores required by

\footnotetext{
${ }^{1}$ In practise performance will decrease slightly when using more than $n_{\mathrm{s}}$ cores, as prefetchers and memory subsystem have to deal with additional memory streams which makes them less efficient; this effect can be observed in Fig. 113.
}

the naive counterpart. Six vs. ten cores being active typically translates into a lower power draw for version using fewer cores ${ }^{2}$ Together with the fact that versions $\left(16 \mathrm{~GB} / b_{\mathrm{s}}\right)$, this results in a better energy-to-solution for the optimized version.

After establishing these facts we propose the following approach to optimize energy consumption: (1) Use the ECM model to guide performance improvements for the single-core implementation. (2) Attempt to lower percore power draw by tuning hardware parameters, e.g. core frequency or COD mode. (3) Never run code with more cores than necessary $\left(n_{\mathrm{s}}\right)$.

\section{THE ECM PERFORMANCE MODEL}

The ECM model 19, 3, 18, 4, 5, is an analytic performance model that, with the exception of sustained memory bandwidth, works exclusively with architecture specifications as inputs. The model estimates the numbers of CPU cycles required to execute a number of iterations of a loop on a single core of a multi- or manycore chip. For multi-core estimates, linear scaling of single-core performance is assumed until a shared bottleneck, such as e.g. main memory bandwidth, is hit. Note that only parts of the model relevant to this work, i.e. the single-core model, are presented here. Readers interested in the full ECM model can find the most recent version for Intel Xeon, Intel Xeon Phi, and IBM POWER8 processors here 6].

The single-core prediction is made up of contributions from the in-core execution time $T_{\text {core }}$, i.e. the time spent executing instructions in the core under the assumption that all data resides in the L1 cache, and the transfer time $T_{\text {data }}$, i.e. the time spent transferring data from its location in the cache/memory hierarchy to the L1 cache. As data transfers in the cache- and memory hierarchy occur at cache line (CL) granularity we chose the number of loop iterations $n_{\text {it }}$ to correspond to one cache line's "worth of work." On Intel architectures, where CLs are $64 \mathrm{~B}$ in size, $n_{\mathrm{it}}=8$ when using double precision (DP) floating-point numbers, because processing eight "doubles" (8 B each) corresponds to exactly one CL worth of work.

Superscalar core designs house multiple execution units, each dedicated to perform certain work: loading, storing, multiplying, adding, etc. The in-core execution time $T_{\text {core }}$ is determined by the unit that takes the longest to retire the instructions allocated to it. Other constraints for the in-core execution time may apply, e.g. the four micro-op per cycle retirement limit of Intel Xeon cores. The model differentiates between core cycles depending on whether data transfers in the cache hierarchy can overlap with in-core execution time. For instance, on Intel Xeons, core cycles in which data is moved between the L1 cache and registers, e.g. cycles

\footnotetext{
${ }^{2}$ In theory it is possible that an implementations using fewer cores to saturate memory bandwidth is less energy-efficient than one that uses more. Consider, for example, an optimization that doubles single-core performance but triples the power drawn by the core. $n_{\mathrm{s}}$ is halved but energy to solution is $50 \%$ higher nonetheless. In practise we have however never observed such a scenario. For all optimizations described in Section 5 the increase in singlecore power draw accompanying an optimization is negligible.
} 
Table 1: Test machine specifications.

\begin{tabular}{|l|c|c|c|c|}
\hline Microarchitecture (Shorthand) & Sandy Bridge-EP (SNB) & Ivy Bridge-EP (IVB) & Haswell-EP (HSW) & Broadwell-EP (BDW) \\
\hline Chip Model & Xeon E5-2680 & Xeon E5-2690 v2 & Xeon E5-2695 v3 & Pre-release \\
\hline Release Date & Q1/2012 & Q3/2013 & Q3/2014 & Q1/2016 \\
\hline non-AVX/AVX Base Freq. & $2.7 \mathrm{GHz} / 2.7 \mathrm{GHz}$ & $3.0 \mathrm{GHz} / 3.0 \mathrm{GHz}$ & $2.3 \mathrm{GHz} / 1.9 \mathrm{GHz}$ & $2.1 \mathrm{GHz} / 2.0 \mathrm{GHz}$ \\
\hline Cores/Threads & $8 / 16$ & $10 / 20$ & $14 / 28$ & $18 / 36$ \\
\hline Latest SIMD Extensions & AVX & AVX & AVX2, FMA3 & AVX2, FMA \\
\hline Core-Private L1/L2 Caches & $8 \times 32 \mathrm{kB} / 8 \times 256 \mathrm{kB}$ & $10 \times 32 \mathrm{kB} / 10 \times 256 \mathrm{kB}$ & $14 \times 32 \mathrm{kB} / 14 \times 256 \mathrm{kB}$ & $18 \times 32 \mathrm{kB} / 18 \times 256 \mathrm{kB}$ \\
\hline Shared Last-Level Cache & $20 \mathrm{MB}(8 \times 2.5 \mathrm{MB})$ & $25 \mathrm{MB}(10 \times 2.5 \mathrm{MB})$ & $35 \mathrm{MB}(14 \times 2.5 \mathrm{MB})$ & $45 \mathrm{MB}(18 \times 2.5 \mathrm{MB})$ \\
\hline Memory Configuration & $4 \mathrm{ch} . \mathrm{DDR} 3-1600$ & $4 \mathrm{ch} . \mathrm{DDR} 3-1866$ & $4 \mathrm{ch} . \mathrm{DDR}-2133$ & $4 \mathrm{ch} . \mathrm{DDR} 4-2133$ \\
\hline Theoretical Mem. Bandwidth & $51.2 \mathrm{~GB} / \mathrm{s}$ & $59.7 \mathrm{~GB} / \mathrm{s}$ & $68.2 \mathrm{~GB} / \mathrm{s}$ & $68.2 \mathrm{~GB} / \mathrm{s}$ \\
\hline Sustained Copy Bandwidth & $39.5 \mathrm{~GB} / \mathrm{s}(77 \%)$ & $43.4 \mathrm{~GB} / \mathrm{s}(74 \%)$ & $50.1 \mathrm{~GB} / \mathrm{s}(73 \%)$ & $51.5(76 \%)$ \\
\hline L1 $\rightarrow$ Reg Bandwidth & $2 \times 16 \mathrm{~B} / \mathrm{cy}$ & $2 \times 16 \mathrm{~B} / \mathrm{cy}$ & $2 \times 32 \mathrm{~B} / \mathrm{cy}$ & $2 \times 32 \mathrm{~B} / \mathrm{cy}$ \\
\hline Reg $\rightarrow$ L1 Bandwidth & $1 \times 16 \mathrm{~B} / \mathrm{cy}$ & $1 \times 16 \mathrm{~B} / \mathrm{cy}$ & $1 \times 32 \mathrm{~B} / \mathrm{cy}$ & $1 \times 32 \mathrm{~B} / \mathrm{cy}$ \\
\hline L1 $\leftrightarrow$ L2 Bandwidth & $32 \mathrm{~B} / \mathrm{cy} \hat{=} 2 \mathrm{cy} / \mathrm{CL}$ & $32 \mathrm{~B} / \mathrm{cy} \hat{=} 2 \mathrm{cy} / \mathrm{CL}$ & $64 \mathrm{~B} / \mathrm{cy} \hat{=} 1 \mathrm{cy} / \mathrm{CL}$ & $64 \mathrm{~B} / \mathrm{cy} \hat{=} \mathrm{cy} / \mathrm{CL}$ \\
\hline L2 $\leftrightarrow \mathrm{L} 3$ Bandwidth & $32 \mathrm{~B} / \mathrm{cy} \hat{=} 2 \mathrm{cy} / \mathrm{CL}$ & $32 \mathrm{~B} / \mathrm{cy} \hat{=} 2 \mathrm{cy} / \mathrm{CL}$ & $32 \mathrm{~B} / \mathrm{cy} \hat{=} 2 \mathrm{cy} / \mathrm{CL}$ & $32 \mathrm{~B} / \mathrm{cy} \hat{=} 2 \mathrm{cy} / \mathrm{CL}$ \\
\hline L3 $\leftrightarrow$ Mem Bandwidth $(\mathrm{copy})$ & $14.5 \mathrm{~B} / \mathrm{cy} \hat{=} 4.4 \mathrm{cy} / \mathrm{CL}$ & $20.0 \mathrm{~B} / \mathrm{cy} \hat{=} 4.4 \mathrm{cy} / \mathrm{CL}$ & $21.7 \mathrm{~B} / \mathrm{cy} \hat{=} 2.9 \mathrm{cy} / \mathrm{CL}$ & $24.6 \mathrm{~B} / \mathrm{cy} \hat{=} 2.6 \mathrm{cy} / \mathrm{CL}$ \\
\hline
\end{tabular}

in which load and/or store instructions are retired, prohibit simultaneous transfer of data between the L1 and L2 cache; these "non-overlapping" cycles contribute to $T_{\text {nOL }}$. Cycles in which no load or store instructions but other instructions, such as e.g. arithmetic instructions, retire are considered "overlapping" cycles and contribute to $T_{\mathrm{OL}}$. The in-core runtime is the maximum of both: $T_{\text {core }}=\max \left(T_{\mathrm{OL}}, T_{\mathrm{nOL}}\right)$.

For modelling data transfers, latency effects are initially neglected, so transfer times are exclusively a function of bandwidth. Cache bandwidths are typically well documented and can be found in vendor data sheets. Depending on how many CLs have to be transferred, the contribution of each level in the memory hierarchy $\left(T_{\mathrm{L} 1 \mathrm{~L} 2}, \ldots, T_{\mathrm{L} 3 \mathrm{Mem}}\right)$ can be determined. Special care has to be taken when dealing with main memory bandwidth, because theoretical memory bandwidth specified in the data sheet and sustained memory bandwidth $b_{\mathrm{s}}$ can differ greatly. Also, in practise $b_{\mathrm{s}}$ depends on the number of load and store streams. It is therefore recommended to empirically determine $b_{\mathrm{s}}$ using a kernel that resembles the memory access pattern of the benchmark to be modeled. Once determined, the time to transfer one CL between the L3 cache and main memory can be derived from the CPU frequency $f$ as $64 \mathrm{~B} \cdot f / b_{\mathrm{s}}$ cycles.

Starting with the Haswell-EP (HSW) microarchitecture, an empirically determined latency penalty $T_{p}$ is applied to off-core transfer times. This departure from the bandwidth-only model has been made necessary by large core counts, the dual-ring design, and separate clock frequencies for core(s) and Uncore all of which increase latencies when accessing off-core data. The penalty is added each time the Uncore interconnect is involved in data transfers. This is the case whenever data is transferred between the L2 and L3 caches, as data is pseudo-randomly distributed between all lastlevel cache segments; and when data is transferred between the L3 cache and memory, because the memory controller is attached to the Uncore interconnect. Instruction times as well as data transfer times, e.g. $T_{\mathrm{L} 1 \mathrm{~L} 2}$ for the time required to transfer data between L1 and L2 caches, can be summarized in shorthand notation: $\left\{T_{\mathrm{OL}} \| T_{\mathrm{nOL}}\left|T_{\mathrm{L} 1 \mathrm{~L} 2}\right| T_{\mathrm{L} 2 \mathrm{~L} 3}+T_{\mathrm{p}} \mid T_{\mathrm{L} 3 \mathrm{Mem}}+T_{\mathrm{p}}\right\}$.

To arrive at a prediction, in-core execution and data transfers times are put together. Depending on whether there exist enough overlapping cycles to hide all data transfers, runtime is given by either $T_{\mathrm{OL}}$ or the sum of non-overlapping core cycles $T_{\text {nOL }}$ plus contributions of data transfers $T_{\text {data }}$, whichever takes longer. $T_{\text {data }}$ consists of all necessary data transfers in the cache/memory hierarchy, plus latency penalties if applicable, e.g. for data coming from the L3 cache: $T_{\text {data }}=T_{\mathrm{L} 1 \mathrm{~L} 2}+T_{\mathrm{L} 2 \mathrm{~L} 3}+$ $T_{\mathrm{p}}$. The prediction is thus $T_{\mathrm{ECM}}=\max \left(T_{\mathrm{OL}}, T_{\mathrm{nOL}}+\right.$ $\left.T_{\text {data }}\right)$. A shorthand notation also exists for the model's prediction: $\left.\left.\left.\left\{T_{\mathrm{ECM}}^{\mathrm{core}}\right\rceil T_{\mathrm{ECM}}^{\mathrm{L} 2}\right\rceil T_{\mathrm{ECM}}^{\mathrm{L} 3}\right\rceil T_{\mathrm{ECM}}^{\mathrm{Mem}}\right\}$.

Converting the prediction from time (in cycles) to performance (work per second) is done by dividing the work per CL $W_{\mathrm{CL}}$ (e.g. floating-point operations, updates, or any other relevant work metric) by the predicted runtime in cycles and multiplying with the processor frequency $f$, i.e. $P_{\mathrm{ECM}}=W_{\mathrm{CL}} / T_{\mathrm{ECM}} \cdot f$.

\section{EXPERIMENTAL TESTBED}

All measurements were performed on standard twosocket Intel Xeon servers. A summary of key specifications of the four generations of processors can be found in Table1. According to Intel's "tick-tock" model, where a "tick" corresponds to a shrink of the manufacturing process technology and a "tock" to a new microarchitecture, IVB and BDW are "ticks" - apart from an increase in core count and a faster memory clock, no major improvements were introduced in these microarchitectures.

HSW, which is a "tock", introduced AVX2, extending the already existing 256 bit SIMD vectorization from floating-point to integer data types. Instructions introduced by the fused multiply-add (FMA) extension are handled by two new, AVX-capable execution units. Data paths between the L1 cache and registers as well as the L1 and L2 caches were doubled. Due to limited scalability of a single ring connecting the cores, HSW chips with more than eight feature a dual-ring design. HSW also introduces the AVX base and maximum AVX Turbo frequencies. The former is the minimum guaranteed frequency when running AVX code on all cores; the latter the maximum frequency when running AVX code on all cores (cf. Table 3 in [9]). Based on workload, the actual frequency varies between this minimum and maximum value. For a more detailed analysis of the 


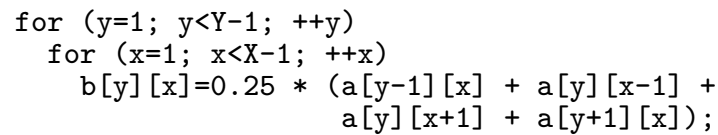

Figure 2: $\mathrm{C}$ implementation for one 2D Jacobi iteration.

differences between the SNB/IVB and HSW microarchitectures see 4 .

For all measurements on SNB and IVB, the CPU frequency was fixed at the nominal $\mathrm{CPU}$ frequency. On HSW and BDW, the CPU frequency was fixed to the non-AVX base frequency ${ }^{3}$ in none of the single-core measurements that feature AVX code could we observe a drop below the non-AVX base frequency. The measured sustained main memory bandwidth displayed in Table 1 is that of the STREAM copy kernel 13] using non-temporal stores, because its memory access pattern corresponds to that of the 2D Jacobi solver. Energy consumption was determined by accessing the running average power limit (RAPL) interface through likwidperfctr 20]. Because node and memory power usage fluctuates depending on node configuration and RAM manufacturer, we chose to only present results pertaining to chip power consumption, i.e. cores, core-private caches, and Uncore (cf. 14.9 in 8]). All code is compiled using the Intel C Compiler version 15.0.2.

\section{SINGLE-CORE OPTIMIZATIONS}

Figure 2 shows the source code for one 2D five-point Jacobi sweep, i.e. the complete update of all grid points. One grid point update computes and stores in $b$ the new state of each point from the values of its four neighbors in a, which holds data from the previous iteration. For results to be representative, the dataset size per socket for all measurements is $16 \mathrm{~GB}$, i.e. each of the two grids is $8 \mathrm{~GB}$ in size and made up of $32768 \times 32768$ doubleprecision numbers. Performance is expressed in "lattice updates per second" (LUP/s), i.e. scalar inner kernel iterations per second.

\subsection{Baseline AVX Implementation}

Using adequate optimization flags (-03 -xHost fno-alias) it is trivial to generate AVX vectorized assembly for the code shown in Figure 2 with recent Intel compilers. This is why we decided to use an AVX vectorized variant instead of scalar code as baseline.

With 256-bit AVX vectorization in place, one CL worth of work (eight LUP/s), consists of eight AVX loads, two AVX stores, six AVX adds, and two AVX multiplication instructions.

To determine data transfers inside the cache hierarchy, we have to examine each load and store in more detail. Storing the newly computed results to array $\mathrm{b}$ involves the transfer of two CLs to/from main memory: because both arrays are too large to fit inside the caches, the store will miss in the L1 cache, triggering a writeallocate of the CL from main memory. After the values in the CL have been updated, the CL will have to be

\footnotetext{
${ }^{3}$ The guaranteed frequency when running code on all cores that does not use AVX instructions.
}

evicted from the caches eventually, triggering another main memory transfer.

The left neighbor $a[y][x-1]$ can always be loaded from the L1 cache since it was used two inner iterations before as right neighbor; access to $a[y+1][x]$ must be loaded from main memory since it was not used before within the sweep.

Based on work by Rivera and Tseng 15. Stengel et al. introduced the layer condition (LC) 18 to help determining where data for $a[y-1][x]$ and $a[y][x+1]$ is coming from. The LC stipulates three successive rows have to fit into a certain cache for accesses to these data to come from this particular cache. Assuming cache $k$ can effectively hold data up to $50 \%$ of its nominal size $C_{k}$, the LC can be formulated as $3 \cdot N \cdot 8 \mathrm{~B}<50 \% \cdot C_{k}$. For $N=32768$, three rows take up $768 \mathrm{kB}$ so on all previously introduced machines (cf. line 8 in Table 1) the LC holds true for the L3 cache.

\section{ECM Model for SNB and IVB}

To process one CL worth of data, eight AVX load, two AVX store, six AVX addition and two AVX multiplication instructions have to executed. Throughput is limited by the two load units. Each load unit has a $16 \mathrm{~B}$ wide data path connecting registers and L1 cache, so retiring a $32 \mathrm{~B}$ AVX load takes two cycles. Using both load units, eight AVX loads take $T_{\mathrm{nOL}}=8 \mathrm{cy}$. Both AVX stores are retired in parallel with the eight loads, so they do not increase $T_{\mathrm{nOL}}$. Computation throughput is limited by the single add port, which takes $T_{\mathrm{OL}}=6 \mathrm{cy}$ to retire all six AVX add instructions. Both AVX multiplications can be processed in parallel with two of the six AVX add instructions.

As established previously, three CLs have to be transferred between L3 and memory: write-allocating and later evicting $\mathrm{b}[\mathrm{y}][\mathrm{x}]$ and loading $\mathrm{a}[\mathrm{y}+1][\mathrm{x}]$. On both SNB and IVB, L3-memory bandwidth is $4.4 \mathrm{cy} / \mathrm{CL}$ (cf. last line in Table 1). This results in $T_{\mathrm{L} 3 \mathrm{Mem}}=13.2 \mathrm{cy}$ for both architectures. The same three CLs have to be transferred between L3 and L2 cache; in addition, CLs for $a[y-1][x]$ and $a[y][x+1]$ have to be transferred from the L3 to the L2 cache. Transferring five CLs at a bandwidth of $2 \mathrm{cy} / \mathrm{CL}$ takes $T_{\mathrm{L} 2 \mathrm{~L} 3}=10 \mathrm{cy}$ on both SNB and IVB. At a L1-L2 bandwidth of 2 cy/CL, moving these five CLs between L2 and L1 cache takes $T_{\mathrm{L} 1 \mathrm{~L} 2}=10 \mathrm{cy}$. Using the ECM short notation to summarize the inputs yields $\{6|| 8|10| 10 \mid 13.2\}$ cy for both SNB and IVB; the corresponding runtime prediction for SNB and IVB is $\{8\rceil 18\rceil 28\rceil 41.2\} \mathrm{cy}$.

For a $2.7 \mathrm{GHz} \mathrm{SNB}$ core the performance prediction is $P_{\mathrm{ECM}}^{M e m}=\frac{8 \mathrm{LUP} / \mathrm{CL}}{41.2 \mathrm{cy} / \mathrm{CL}} \cdot 2.7 \mathrm{GHz}=524 \mathrm{MLUP} / \mathrm{s}$. For IVB the model predicts a performance of $582 \mathrm{MLUP} / \mathrm{s}$.

\section{ECM Model for HSW and BDW}

On HSW and BDW, the address generation units (AGUs) are the bottleneck for $T_{\mathrm{nOL}}$. Each load/store instruction accesses an AGU to compute the referenced memory address. With only two AGUs capable of performing the required addressing operations available, retiring all ten load/store instructions takes $T_{\mathrm{nOL}}=$ $5 \mathrm{cy}$. HSW and BDW posses a single AVX add unit 
just like $\mathrm{SNB}$ and IVB, so $T_{\mathrm{OL}}=6 \mathrm{cy}$.

The off-core latency penalty was empirically estimated at approximately 1.6 cycles for both HSW and BDW and is applied per CL transfer that takes place over the Uncore interconnect, i.e. all transfers between L3 and L2 caches as well as transfers between memory and the L3 cache. The effective L3-Mem bandwidth is thus $2.9+1.6 \mathrm{cy}$ on HSW and $2.6+1.6 \mathrm{cy}$ on BDW; the effective L2-L3 is $2+1.6$ cy on HSW and $2+1.6$ cy on BDW.

Transferring the three required CLs then results in $T_{\mathrm{L} 3 \mathrm{Mem}}=8.7+4.8 \mathrm{cy}$ on $\mathrm{HSW}$ resp. $T_{\mathrm{L} 3 \mathrm{Mem}}=$ $7.8+4.8 \mathrm{cy}$ on BDW. Moving five CLs between the L2 and L3 caches takes $T_{\mathrm{L} 2 \mathrm{~L} 3}=10+8 \mathrm{cy}$ on both HSW and BDW. At a L1-L2 bandwidth of $1 \mathrm{cy} / \mathrm{CL}$, moving the same five CLs takes $T_{\mathrm{L} 1 \mathrm{~L} 2}=5 \mathrm{cy}$ on both microarchitectures. The ECM inputs are thus $\{6|| 5|5| 10+8 \mid 8.7+4.8\}$ cy for HSW and for BDW $\{6 \| 5|5| 10+8 \mid 7.8+4.8\}$ cy. The corresponding runtime predictions are $\{6\rceil 10\rceil 28\rceil 41.5\}$ cy for HSW and $\{6\rceil 10\rceil 28\rceil 40.6\}$ cy for BDW. The performance predictions are $443 \mathrm{MLUP} / \mathrm{s}$ for HSW and $413 \mathrm{MLUP} / \mathrm{s}$ for BDW.

\subsection{Cache Blocking Optimization}

One way to increase the performance of the singlecore implementation is to reduce the amount of time spent transferring data inside the cache hierarchy. As previously established, $\mathrm{a}[\mathrm{y}-1][\mathrm{x}]$ and $\mathrm{a}[\mathrm{y}][\mathrm{x}+1]$ are loaded from the L3 cache, because the L1 and L2 caches are too small to fulfill the LC for $N=32768$. Using cache blocking, it is possible to enforce the LC in arbitrary cache levels. This is done by partitioning the grid into stripes along the $y$-axis; the grid is then processed stripe by stripe. The diameter of the stripes, also known as blocking factor $b_{x}$, is chosen is such a way that the LC is met for a given cache level. If L2 blocking is desired, the $\mathrm{L} 2$ cache size of $256 \mathrm{kB}$ requires that $b_{x}$ should be chosen smaller than 5461 .

Efficient blocking for the $32 \mathrm{kB} \mathrm{L1}$ cache not as straightforward. Although determining $b_{x}<682$ is simple using the LC, naive L1 blocking in $x$-direction has negative side effects. With most of the data for one CL update coming from L1, the L2 cache is less busy. This slack is detected by hardware prefetchers, making them more aggressive, leading to data being prefetched from main memory. With $b_{x} \approx 680$, the size of one stripe is $680 \cdot 32768 \cdot 8 \mathrm{~B}=170 \mathrm{MB}$ - too large for the L3 cache, which means that data prefetched from main memory will be preempted from the cache before it is used. This can be avoided either by disabling some of the prefetchers or additionally blocking in $y$-direction. We used the latter, because disabling prefetchers might degrade performance elsewhere. To guarantee data is used before being preempted, the size of each chunk should be chosen smaller than $50 \%$ of a single L3 segment $]^{4}$ e.g. $b_{y}<50 \% \cdot 2.5 \mathrm{MB} /\left(b_{x} \cdot 8 \mathrm{~B}\right)$.

\footnotetext{
${ }^{4}$ In the multi-core scenario, all cores might be active and store data in the shared L3 cache; the capacity dedicated to a single core is thus that of a single L3 segment adjusted by $50 \%$ to reflect effective cache size.
}

\section{ECM Model for SNB and IVB}

Other than causing negligible loop overhead cache blocking does not change the instructions that have to be retired to process one CL; thus $T_{\mathrm{OL}}$ and $T_{\mathrm{nOL}}$ remain unchanged.

L2 blocking reduces the number of CLs transferred between L3 and L2 from five to three, lowering $T_{\mathrm{L} 2 \mathrm{~L} 3}$ from ten to six cycles on both SNB and IVB. The runtime prediction $T_{\mathrm{ECM}}^{\mathrm{Mem}}$ for both SNB and IVB is reduced from 41.2 to $37.2 \mathrm{cy}$, leading to a performance prediction $P_{\mathrm{ECM}}^{\mathrm{Mem}}$ of $580 \mathrm{MLUP} / \mathrm{s}$ for SNB and $645 \mathrm{MLUP} / \mathrm{s}$ for IVB.

Similarly L1 blocking reduces the CL traffic between $\mathrm{L} 2$ and L1 caches from five to three CLs, lowering $T_{\mathrm{L} 1 \mathrm{~L} 2}$ from ten to six cycles on both SNB and IVB. Again, the runtime prediction $T_{\mathrm{ECM}}^{\mathrm{Mem}}$ for both microarchitectures is reduced by four cycles from 37.2 to $33.2 \mathrm{cy}$. The predicted performance $P_{\mathrm{ECM}}^{\mathrm{Mem}}$ increases to $651 \mathrm{MLUP} / \mathrm{s}$ on SNB and $723 \mathrm{MLUP} / \mathrm{s}$ on IVB.

\section{ECM Model for HSW and BDW}

The effect of L2 blocking is more pronounced on HSW and BDW, because the cost of transferring a CL is higher on these microarchitectures due to the latency penalty. L2 blocking lowers $T_{\mathrm{L} 2 \mathrm{~L} 3}$ from $10+8$ to $6+4.8 \mathrm{cy}$ on HSW and BDW. In turn, the runtime prediction $T_{\mathrm{ECM}}^{\mathrm{Mem}}$ for $\mathrm{HSW}$ is reduced from 41.5 to $34.3 \mathrm{cy}$ and from 40.6 to $33.4 \mathrm{cy}$ on BDW. The performance prediction $P_{\mathrm{ECM}}^{\mathrm{Mem}}$ increases to $536 \mathrm{MLUP} / \mathrm{s}$ on HSW and $503 \mathrm{MLUP} / \mathrm{s}$ on BDW.

Because the L1-L2 bandwidth increased from 2 cy/CL on SNB/IVB to $1 \mathrm{cy} / \mathrm{CL}$ on $\mathrm{HSW} / \mathrm{BDW}$, the performance improvement offered by L1 blocking is less pronounced than on SNB and IVB. With the number of CL transfers lowered from five to three with L1 blocking, $T_{\mathrm{L} 1 \mathrm{~L} 2}$ is reduced from five to three cycles. The runtime prediction $T_{\mathrm{ECM}}^{\mathrm{Mem}}$ for $\mathrm{HSW}$ is reduced from 34.3 to $32.3 \mathrm{cy}$; on BDW from 33.4 to $31.4 \mathrm{cy}$. The predicted performance $P_{\mathrm{ECM}}^{\mathrm{Mem}}$ is increased to $570 \mathrm{MLUP} / \mathrm{s}$ on HSW and $535 \mathrm{MLUP} / \mathrm{s}$ on BDW.

\subsection{Cluster-on-Die Mode}

As a workaround to the limited scalability of the physical ring interconnect introduced with Westmere-EX, HSW and BDW switch to a dual-ring design. HSW uses the so-called "eight plus $x$ " design, in which the first eight cores of a chip are attached to a primary ring and the remaining cores (six for the model introduced in Section 44) are attached to a secondary ring; BDW uses a symmetric design. Two queues enable data to pass between rings. In the default (non-COD) mode, the physical topology is hidden from the operating system, i.e. all cores are exposed within the same non-uniform memory access (NUMA) domain.

To understand the latency problems caused by the interconnect, we examine the route data travels inside the Uncore. Using a hashing function data is is distributed across all L3 segments based on its memory address. When accessing data in the L3 cache, there is a high probability it must be fetched from remote L3 segments. In the worst case, this is a segment on the other ring so there might be a high latency involved. In the case of a 
L3 miss the situation gets worse. Each physical ring has attached to it a memory controler (MC) and the choice which MC to use is again based on the data's address. So a L3 miss in a segment on one physical ring does not imply that this ring's MC will be used to fetch the data from memory. That leads to cases in which a large number of hops and multiple cross-physical ring transfers are involved when getting data from main memory.

One way to reduce these latencies is the new COD mode introduced together with the dual-ring design on HSW and BDW in which cores are separated into two physical clusters of equal size. The latency reduction is achieved by adapting the involved hashing functions. Data requested by a core of a cluster will only be placed in the cluster's L3 segments; in addition, all memory transfers are routed to the MC dedicated to the cluster. Thus, for NUMA-aware codes, COD mode effectively lowers the latency by reducing the diameter and the mean distance of the dual-ring interconnect by restricting each cluster to its own physical ring. For a more detailed analysis of COD mode see 4. On the HSW chip used for benchmarks, COD mode lowers the interconnect latency by $0.5 \mathrm{cy}$; on the employed BDW chip, where a single ring still has eleven cores attached to it, the latency is only reduced by $0.3 \mathrm{cy}$.

\section{ECM Model for HSW and BDW}

With COD enabled, the per-CL Uncore latency penalty is reduced to $1.1 \mathrm{cy}$ on HSW resp. $1.3 \mathrm{cy}$ on BDW. This leads to $T_{\mathrm{L} 3 \mathrm{Mem}}=8.7+3.3 \mathrm{cy}$ on HSW resp. $7.8+3.9 \mathrm{cy}$ on BDW. Because the Uncore is also involved in L2-L3 transfers, the latency improvement caused by enabling COD also positively influences $T_{\mathrm{L} 2 \mathrm{~L} 3}$. To transfer three CLs, $6+3.3 \mathrm{cy}$ are required on HSW resp. $6+3.9$ cy on BDW. The resulting ECM inputs are $\{6 \| 5|3| 6+3.3 \mid 8.7+3.3\}$ for HSW and $\{6 \| 5|3| 6+3.9 \mid 7.8+3.9\}$ for BDW. The corresponding runtime prediction is $\{6\rceil 8\rceil 17.3\rceil 29.3\}$ on HSW and $\{6\rceil 8\rceil 17.9\rceil 29.6\}$ on BDW. The performance predicted by the ECM model is $628 \mathrm{MLUP} / \mathrm{s}$ for HSW and $567 \mathrm{MLUP} / \mathrm{s}$ on BDW.

\subsection{Non-Temporal Stores}

Streaming or non-temporal (NT) stores are special instructions that avoid write-allocates on modern Intel microarchitectures. Without NT stores, storing the newly computed result $\mathrm{b}[\mathrm{y}][\mathrm{x}]$ triggers a writeallocate. The old data is brought in from memory and travels through the whole cache hierarchy. Thus the first benefit of using NT stores is that the unnecessary transfer of $\mathrm{b}[\mathrm{y}][\mathrm{x}]$ from memory to the L1 cache no longer takes place. In addition, NT stores will also strip some cycles off the time involved getting the new result to main memory. Using regular stores, the newly computed result is written to the L1 cache, from where the data has to be evicted at some point through the whole cache hierarchy into memory. Using NT stores, CLs are sent via the $\mathrm{L} 1$ cache to the line fill buffers (LFBs); from there, they are transfered directly to memory and do not pass through the L2 and L3 caches. Although the benefits should apply equally to all microarchitectures, there are shortcomings in SNB and IVB that make single-core implementations using NT stores slower than their regular stores counterpart. The positive impact of NT stores can only be leveraged in multi-core scenarios on these microarchitectures, which is why we chose to omit ECM models and measurements for the NT store implementation for SNB and IVB.

\section{ECM Model for HSW and BDW}

Transferring $a[y+1][x]$ between the L1 and L2 caches takes 1 cy. Although the transfer is not strictly between the L1 and L2 cache, the cycle spent transferring the CL for $\mathrm{b}[\mathrm{y}][\mathrm{x}]$ from the $\mathrm{L} 1$ cache to the line fill buffer (LFB) is booked in $T_{\mathrm{L} 1 \mathrm{~L} 2}$ as well, making for a total L1-L2 transfer time of 2 cy. Loading a $[y+1][x]$ from the L3 to the L2 cache takes $T_{\mathrm{L} 2 \mathrm{~L} 3}=2+1.1 \mathrm{cy}$ on HSW and $2+1.3$ cy on BDW. Getting the CL containing $\mathrm{a}[\mathrm{y}+1][\mathrm{x}]$ from memory takes $2.9+1.1 \mathrm{cy}$ on HSW and $2.6+1.3 \mathrm{cy}$ on BDW; again, although the transfer of $b[y][x]$ is strictly not between the L3 cache and memory, the transfer time to send the CL from the LFB to memory is booked in $T_{\mathrm{L} 3 \mathrm{Mem}}$, making for a total L3-Mem transfer time of $5.8+2.2 \mathrm{cy}$ on HSW and $5.2+2.6 \mathrm{cy}$ on BDW. In summary, the full ECM inputs are $\{6 \| 5|2| 2+1.1 \mid 5.8+2.2\}$ cy on HSW and $\{6 \| 5|2| 2+1.3 \mid 5.2+2.6\}$ cy on BDW; the corresponding ECM runtime prediction is $\{6\rceil 7\rceil 10.1\rceil 18.1\}$ on HSW and $\{6\rceil 7\rceil 10.3\rceil 18.1\}$. The in-memory performance prediction by the ECM model is $P_{\mathrm{ECM}}^{\mathrm{Mem}}=$ $1016 \mathrm{MLUP} / \mathrm{s}$ on HSW and $928 \mathrm{MLUP} / \mathrm{s}$ on BDW.

\section{SINGLE-CORE RESULTS}

Table 2 contains a summary of the ECM inputs and predictions discussed in Section 5 , as well as measurements of performance, power, and energy consumption for one 2D Jacobi iteration using a $16 \mathrm{~GB}$ dataset. The model correctly predicts performance with a mean error of $3 \%$ and a maximum error of $7 \%$, which indicates that all single-core performance engineering measures work as intended. On SNB and IVB performance increases of around $1.3 \times$ are achieved; on HSW with $2.2 \times$ resp. BDW with $2.1 \times$ improvement the increases are even more pronounced. Measurements obtained via the RAPL interface indicate increases in power consumption due to optimizations are negligible (in the range of $2 \%$ ). With power draw almost constant, this means that the performance gains directly translate into energy improvements.

An interesting observation regarding single-core power consumption 5 can be made when comparing the different microarchitectures that can be explained using Intel's "tick-tock" model. Power decreases with "ticks," i.e. a shrink in manufacturing size and the accompanying decreases in dynamic power; power increases with "tocks," i.e. major improvements in microarchitecture: The "tick" from SNB which uses $32 \mathrm{~nm}$ to IVB which uses $22 \mathrm{~nm}$ technology corresponds to a $10 \%$ decrease in power consumption. HSW, the only "tock" in Table 2 uses the same $22 \mathrm{~nm}$ process as IVB but introduced

\footnotetext{
${ }^{5}$ The RAPL counters can not report power consumption of individual cores but only that of the whole package. Thus reported values also include power drawn by Uncore facilities, e.g. all L3 segments and the interconnect.
} 
Table 2: Summary of ECM inputs, ECM predictions, as well as measured performance, power consumption, and energy-tosolution for one 2D Jacobi iteration with a dataset size of 16 GB.

\begin{tabular}{|c|c|c|c|c|c|c|c|c|c|c|c|c|c|}
\hline parch & Version & \multicolumn{5}{|c|}{$\begin{array}{c}\text { ECM } \\
\text { input }[\mathrm{cy}]\end{array}$} & \multicolumn{3}{|c|}{$\begin{array}{l}\text { ECM pre- } \\
\text { diction }[\mathrm{cy}\end{array}$} & $\begin{array}{c}P_{\mathrm{ECM}}^{\mathrm{Mem}} \\
{[\mathrm{MLUP} / \mathrm{s}]}\end{array}$ & $\begin{array}{l}\text { Measured } \\
{[\mathrm{MLUP} / \mathrm{s}]}\end{array}$ & $\begin{array}{c}\text { Chip } \\
\text { Power }[\mathrm{W}]\end{array}$ & $\begin{array}{l}\text { Chip Energy- } \\
\text { to-Solution [J] }\end{array}$ \\
\hline \multirow[t]{3}{*}{ SNB } & Baseline & & $\{6$ & & \begin{tabular}{|l|l|l|}
8 & 10 & 10
\end{tabular} & $13.2\}$ & $\{8$ & 8]18]28]. & $41.2\}$ & 524 & 514 & 35.9 & 75.0 \\
\hline & L2 blocked & & $\{6$ & & $8|10| \mathbf{6} \mid$ & $13.2\}$ & $\{8$ & $18] \mathbf{2 4}$ & $37.2\}$ & 580 & 623 & 36.4 & 62.7 \\
\hline & L1 blocked & & & & $\| 8|\mathbf{6}| 6 \mid$ & $13.2\}$ & $\{8$ & 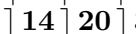 & 33.2 & 651 & 672 & 36.6 & 58.4 \\
\hline \multirow[t]{3}{*}{ IVB } & Baseline & & $\{6$ & & \begin{tabular}{|l|l|l|}
8 & 10 & 10
\end{tabular} & $13.2\}$ & $\{8$ & \begin{tabular}{|l|l|l|}
3 & 18 & 28
\end{tabular} & $41.2\}$ & 582 & 539 & 32.7 & 65.3 \\
\hline & L2 blocked & & $\{6$ & & $8|10| 6 \mid$ & $13.2\}$ & $\{8$ & $187 \mathbf{2 4}$ & $37.2\}$ & 645 & 651 & 34.1 & 56.1 \\
\hline & L1 blocked & & & & $\mid \begin{array}{l}8|6| 6 \mid \\
6\end{array}$ & $13.2\}$ & $\{8$ & 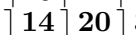 & 33.2 & 722 & 714 & 33.0 & 49.6 \\
\hline \multirow[t]{5}{*}{ HSW } & Baseline & $\{6$ & 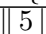 & 5 & $\mid 10+8$ & $8.7+4.8\}$ & $\{6$ & \begin{tabular}{|l|l|l|}
5 & 10 & 28 \\
\end{tabular} & $41.5\}$ & 443 & 435 & 50.9 & 125.6 \\
\hline & L2 blocked & $\{6$ & $|5|$ & & $|6+4.8|$ & $\{8.7+4.8\}$ & $\{6\rceil$ & $10\rceil \mathbf{2 0 . 8}$ & 734.3 & 536 & 529 & 51.0 & 103.5 \\
\hline & L1 blocked & $\{6$ & 5 & 3 & $|6+4.8|$ & $8.7+4.8\}$ & $\{6$ & $8] 18.8$ & 32.3 & 570 & 579 & 52.1 & 96.6 \\
\hline & L1 b.+CoD & $\{6$ & 5 & & $|6+\mathbf{3 . 3}|$ & $\{8.7+\mathbf{3 . 3}\}$ & $\{6$ & $8\rceil 17.3\rceil$ & $29.3\}$ & 628 & 625 & 51.3 & 88.0 \\
\hline & $\mathrm{L} 1 \mathrm{~b} .+\mathrm{CoD}+\mathrm{nt}$ & $\{6$ & $5 \mid$ & 2 & $|2+1.1|$ & $5.8+2.2\}$ & $\{6$ & 710.1 & 18.1 & 1016 & 951 & 51.0 & 57.6 \\
\hline \multirow[t]{5}{*}{ BDW } & Baseline & $\{6$ & 5 & 5 & $|10+8|$ & $7.8+4.8\}$ & $\{6$ & \begin{tabular}{|c|c|c|}
5 & 10 & 28
\end{tabular} & $40.6\}$ & 413 & 407 & 44.2 & 116.5 \\
\hline & L2 blocked & $\{6$ & $|5|$ & & $|6+4.8|$ & $\{7.8+4.8\}$ & $\{6\rceil$ & $10\rceil \mathbf{2 0 . 8}$ & 133.4 & 503 & 489 & 44.3 & 97.4 \\
\hline & L1 blocked & $\{6$ & 5 & 3 & $6+4.8 \mid$ & $7.8+4.8\}$ & $\{6$ & $8] 18.8$ & 31.4 & 535 & 509 & 44.5 & 93.7 \\
\hline & L1 b.+CoD & $\{6 \mid$ & $51:$ & 3 & $|6+\mathbf{3 . 9}|$ & $7.8+\mathbf{3 . 9}\}$ & $\{6$ & $8\rceil 17.9]$ & $29.6\}$ & 567 & 561 & 44.6 & 86.0 \\
\hline & $\mathrm{L} 1$ b. $+\mathrm{CoD}+\mathrm{nt}$ & $\{6$ & $|5|$ & 2 & $|2+1.3|$ & $5.2+2.6$ & $\{6$ & 710.3 & $18.1\}$ & 928 & 862 & 45.4 & 56.6 \\
\hline
\end{tabular}

major improvements in the microarchitecture (cf. Section 4) which lead to a more than $50 \%$ higher power draw. BDW, a "tick" which uses a $14 \mathrm{~nm}$ process uses $11 \%$ less power than HSW. While it is tempting to generalize from these results, note that the reported numbers are specific to the 2D Jacobi application and the used chip models. We also believe that while a surge in power consumption occurs with the HSW "tock" the cause to be a combination of microarchitectural improvements and the higher core count ${ }^{6}$

\section{CHIP-LEVEL OBSERVATIONS}

Although the ECM model can be used to predict multi-core scaling behavior $6,4,18$, due to space constraints chip-level discussion is restricted to empirical results. The graphs in Figure 3 show the improvements discussed in Section 5 and relate measured performance and energy-to-solution for different core counts 7 IVB (Fig. 3a) and HSW (Fig. 3b) are chosen as representatives to demonstrate that the different optimizations have different impacts on performance and energy consumption depending on the microarchitecture.

On IVB, running on $n_{\mathrm{s}}=5$ instead of ten cores reduces energy consumption by $13 \% 44.4 \mathrm{~J}$ to $34.8 \mathrm{~J}$; on HSW, running on seven instead of 14 cores amounts to a reduction of $16 \%$ from $57.0 \mathrm{~J}$ to $47.6 \mathrm{~J}$. The positive impacts of L1 blocking can be observed for both microarchitectures, corresponding to a further decrease of energy consumption by $16 \%$ to $29.1 \mathrm{~J}$ on IVB resp. $10 \%$ to $43.0 \mathrm{~J}$ on HSW. COD mode brings energy consumption on HSW further down by $6 \%$ to $40.5 \mathrm{~J}$.

When it comes to NT stores the differences in the microarchitectures become visible. On IVB, the per-core performance of the implementation using L1 blocking and NT stores (dark blue line in Fig. 3a) is lower than that of the version using regular stores. A version using

\footnotetext{
${ }^{6}$ Despite only one core being active in the measurements, all L3 cache segments are active and draw power; a chip with more cores will thus draw more power in single-core use cases.

${ }^{7}$ The leftmost measuring point of each graph corresponds to one core. Following the line attached to a point to the next corresponds to one more core being active. For demonstration purposes the purple graph in Fig. 3a has some core counts annotated.
}

NT stores without L1 blocking (bright blue line) does not even manage to sustain memory bandwidth. Due to this bad per-core performance, NT stores on SNB/IVB have almost no positive impact on energy consumption! On HSW, per-core performance as expected is exactly $1.5 \times$ faster with NT stores, enabling a lowering in energy consumption by $33 \%$ to $27.0 \mathrm{~J}$.

Another difference in microarchitecture surfaces when examining frequency-tuning for potential energy savings. Before HSW, i.e. on SNB and IVB, the chip's Uncore frequency was set to match the frequency of the fastest active core. Because the Uncore contains L3 segments, ring interconnect, and memory controllers, L3 and memory bandwidth is a function of core frequency on SNB/IVB. This effect can be observed when setting the frequency to $1.2 \mathrm{GHz}$ on IVB (magenta line in Fig 3a): Despite providing the best energy-to-solution, performance is hurt badly. On HSW, the Uncore is clocked independently. This leads to a situation in which (due to frequency-induced lower per-core performance) more cores are required to saturate main memory bandwidth (cf. magenta line in Fig 3 p); however, the lower per-core power consumption translates into overall energy savings of $11 \%$, lowering energy-tosolution to $24.0 \mathrm{~J}$. These results indicate that energy consumption could be further reduced if the chips offered frequencies below their current $1.2 \mathrm{GHz}$ floor.

Table 3 contains a summary of the final result pertaining to energy saving for each microarchitecture. The "reference" value corresponds to energy consumption of the baseline implementation running on all cores clocked at the chip's nominal frequency. Energy-tosolution of the most energy-efficient version is listed in the "optimized" column along with the number of active cores and their frequency that was used to obtain the results in the "configuration" column.

\section{CONCLUSION}

We have applied a new energy-optimization approach to a 2D Jacobi solver and analyzed its effects on a range of recent Intel multi-core chips. Using the executioncache-memory (ECM) model single-core software improvements were described and their accuracy validated 

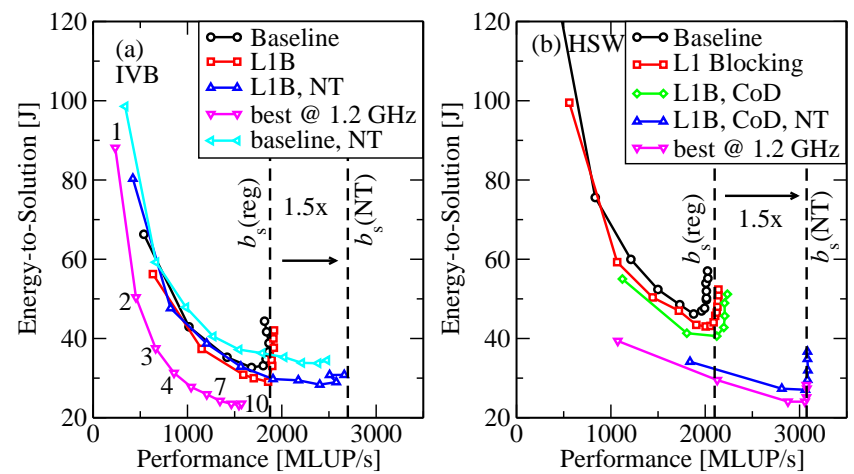

Figure 3: Performance vs. energy-to-solution for different core counts on IVB (a) and HSW (b).

by measurements. For the first time, the ECM model has been (a) extended to incorporate non-temporal (NT) stores and (b) applied to a Broadwell-EP chip. We found energy consumption can reduces by a factor of $2.1 \times$ on Sandy Bridge-EP, $2.0 \times$ on Ivy Bridge-EP, $2.4 \times$ on Haswell-EP, and $2.3 \times$ on Broadwell-EP. Further, we found that while NT stores can increase performance on Sandy- and Ivy Bridge-based E5 processors, a direct positive impact on energy consumption could not be observed; only in combination with frequency tuning do NT stores offer a better energy-to-solutions on these architectures. Measurements indicate that this problem has been solved on Haswell-EP and Broadwell-EP. Moreover, our results indicate that future microarchitectures that keep core and Uncore frequencies decoupled could offer improved energy-efficiency if core frequencies below the current $1.2 \mathrm{GHz}$ floor were available. Beyond these immediate results we have demonstrated the viability of our energy-optimization approach.

\section{REFERENCES}

[1] M. Curtis-Maury, F. Blagojevic, C. Antonopoulos, and D. Nikolopoulos. Prediction-based power-performance adaptation of multithreaded scientific codes. Parallel and Distributed Systems, IEEE Transactions on, 19(10):1396-1410, Oct 2008.

[2] Y. Dong, J. Chen, X. Yang, L. Deng, and X. Zhang. Energy-oriented openmp parallel loop scheduling. In Parallel and Distributed Processing with Applications, 2008. ISPA '08. International Symposium on, pages 162-169, Dec 2008.

[3] G. Hager, J. Treibig, J. Habich, and G. Wellein. Exploring performance and power properties of modern multicore chips via simple machine models. Concurrency Computat.: Pract. Exper., 2013. DOI: 10.1002/cpe.3180.

[4] J. Hofmann, D. Fey, J. Eitzinger, G. Hager, and G. Wellein. Architecture of Computing Systems - ARCS 2016: 29th International Conference, Nuremberg, Germany, April 4-7, 2016, Proceedings, chapter Analysis of Intel's Haswell Microarchitecture Using the ECM Model and Microbenchmarks, pages 210-222. Springer International Publishing, Cham, 2016.

[5] J. Hofmann, D. Fey, M. Riedmann, J. Eitzinger, G. Hager, and G. Wellein. Parallel Processing and Applied Mathematics: 11th International Conference, PPAM 2015, Krakow, Poland, September 6-9, 2015. Revised Selected Papers, Part I, chapter Performance Analysis of the Kahan-Enhanced Scalar Product on Current Multicore Processors, pages 63-73. Springer International Publishing, Cham, 2016.

[6] J. Hofmann, D. Fey, M. Riedmann, J. Eitzinger, G. Hager, and G. Wellein. Performance analysis of the
Table 3: Summary of chip-level benchmarks. Energy improvements shown in parenthesis.

\begin{tabular}{|c|c|c|c|}
\hline uarch & Reference & Optimized & Configuration \\
\hline SNB & $58.7 \mathrm{~J}$ & $28.0 \mathrm{~J}(2.1 \times)$ & 7 cores, $1.2 \mathrm{GHz}$ \\
\hline IVB & $44.4 \mathrm{~J}$ & $22.3 \mathrm{~J}(2.0 \times)$ & 9 cores, $1.5 \mathrm{GHz}$ \\
\hline HSW & $57.0 \mathrm{~J}$ & $24.0 \mathrm{~J}(2.4 \times)$ & 4 cores, $1.2 \mathrm{GHz}$ \\
\hline BDW & $47.9 \mathrm{~J}$ & $20.4 \mathrm{~J}(2.3 \times)$ & 4 cores, $1.2 \mathrm{GHz}$ \\
\hline
\end{tabular}

kahan-enhanced scalar product on current multi-corecore and many-core processors. Concurrency and Computation: Practice and Experience, pages n/a-n/a, 2016. cpe.3921.

[7] F. I. in Cooperation with Fraunhofer ISI. Abschätzung des Energiebedarfs der weiteren Entwicklung der Informationsgesellschaft, 2009.

[8] Intel Corp. Intel 64 and IA-32 Architectures Software Developer's Manual, 2016. Version: April 2016.

[9] Intel Corp. Intel Xeon Processor E5 v3 Product Family: Processor Specification Update, 2016. Version: February 2016.

[10] J. Koomey. Growth in data center electricity use 2005 to 2010. Oakland, CA: Analytics Press. August, 1:2010, 2011.

[11] D. Li, B. R. de Supinski, M. Schulz, D. S. Nikolopoulos, and K. W. Cameron. Strategies for energy-efficient resource management of hybrid programming models. IEEE Trans. Parallel Distrib. Syst., 24(1):144-157, 2013.

[12] A. Mazouz, A. Laurent, B. Pradelle, and W. Jalby. Evaluation of cpu frequency transition latency. Computer Science - Research and Development, 29(3):187-195, 2013

[13] J. D. McCalpin. Memory bandwidth and machine balance in current high performance computers. IEEE Computer Society Technical Committee on Computer Architecture (TCCA) Newsletter, pages 19-25, Dec. 1995.

[14] A. Miyoshi, C. Lefurgy, E. Van Hensbergen, R. Rajamony, and R. Rajkumar. Critical power slope: Understanding the runtime effects of frequency scaling. In Proceedings of the 16th International Conference on Supercomputing, ICS '02, pages 35-44, New York, NY, USA, 2002. ACM.

[15] G. Rivera and C.-W. Tseng. Tiling optimizations for 3d scientific computations. In Proceedings of the 2000 ACM/IEEE Conference on Supercomputing, SC '00, Washington, DC, USA, 2000. IEEE Computer Society.

[16] B. Rountree, D. Lowenthal, S. Funk, V. W. Freeh, B. de Supinski, and M. Schulz. Bounding energy consumption in large-scale mpi programs. In Supercomputing, 2007. SC '07. Proceedings of the 2007 ACM/IEEE Conference on, pages 1-9, Nov 2007.

[17] B. Rountree, D. K. Lownenthal, B. R. de Supinski, M. Schulz, V. W. Freeh, and T. Bletsch. Adagio: Making dvs practical for complex hpc applications. In Proceedings of the 23rd International Conference on Supercomputing, ICS '09, pages 460-469, New York, NY, USA, 2009. ACM.

[18] H. Stengel, J. Treibig, G. Hager, and G. Wellein. Quantifying performance bottlenecks of stencil computations using the Execution-Cache-Memory model. In Proceedings of the 29th ACM International Conference on Supercomputing, ICS '15, New York, NY, USA, 2015. ACM.

[19] J. Treibig and G. Hager. Introducing a performance model for bandwidth-limited loop kernels. In R. Wyrzykowski, J. Dongarra, K. Karczewski, and J. Wasniewski, editors, Parallel Processing and Applied Mathematics, volume 6067 of Lecture Notes in Computer Science, pages 615-624. Springer Berlin / Heidelberg, 2010.

[20] J. Treibig, G. Hager, and G. Wellein. Likwid performance tools. In C. B. et al., editor, Competence in High Performance Computing 2010, pages 165-175. Springer Berlin Heidelberg, 2012.

[21] C.-J. Wu and M. Martonosi. Characterization and dynamic mitigation of intra-application cache interference. In Proceedings of the IEEE International Symposium on Performance Analysis of Systems and Software, ISPASS '11, pages 2-11, Washington, DC, USA, 2011. IEEE Computer Society. 